\title{
Diagnostic usefulness of myocardial perfusion imaging in patients reluctant to undergo angiography
}

This article was published in the following Dove Press journal:

Research Reports in Clinical Cardiology

I8 April 2016

Number of times this article has been viewed

\author{
Akhil Narang \\ Amita Singh \\ Amit R Patel \\ Department of Medicine, Section of \\ Cardiology, University of Chicago, \\ Chicago, IL, USA
}

\begin{abstract}
Patients with known or suspected coronary artery disease are often referred for ischemic testing to aid in risk assessment and guide management when symptoms develop. Invasive coronary angiography and percutaneous intervention are typically reserved for patients with symptoms refractory to medical management. The use of noninvasive modalities with myocardial perfusion imaging is a powerful diagnostic and prognostic tool for patients reluctant to undergo angiography. This review focuses on evaluation of coronary artery disease with myocardial perfusion imaging using single-photon emission computerized tomography, positron emission tomography, myocardial contrast echocardiography, cardiovascular magnetic resonance, and cardiovascular computerized tomography.
\end{abstract}

Keywords: noninvasive, myocardial perfusion imaging, coronary artery disease, stable angina

\section{Introduction}

Cardiovascular disease remains the leading cause of death in the USA with nearly one in three deaths. ${ }^{1,2}$ After the age of 40 years, nearly half of men and one-third of women develop coronary artery disease (CAD). ${ }^{3,4}$ Typically, atherosclerotic disease leading to obstructive CAD takes decades to develop. Patients are often asymptomatic for years. However, when symptoms of angina or angina equivalent manifest, patients are referred for myocardial perfusion imaging (MPI) not only for risk stratification but also to determine if they might benefit from revascularization. As with most diagnostic tests, MPI is most valuable for patient management when they have an intermediate pretest probability of CAD. ${ }^{5}$ In addition, the use of invasive coronary angiography has not been shown to be a cost-effective first-line testing strategy when the pretest probability is $<75 \%$. ${ }^{6}$ In fact, a significant proportion of patients referred for coronary angiography are determined to have no significant obstructive coronary disease. ${ }^{7}$

While diagnostic coronary angiography is typically very safe with the rate of major complication rate $<1 \%,{ }^{8}$ patients at times prefer a noninvasive approach when offered both options. In one study of 111 consecutive patients with suspected CAD, there was greater preference for noninvasive testing (either cardiovascular computerized tomography $[\mathrm{CCT}]$ or cardiovascular magnetic resonance $[\mathrm{CMR}]$ ) due to increased comfort and less pain when compared to invasive coronary angiography. ${ }^{9}$

When selecting a noninvasive imaging modality for evaluation of suspected CAD, a variety of options exist. Historically, single-photon emission computerized tomography (SPECT) and myocardial contrast echocardiography (MCE) have been widely
Correspondence:Amit R Patel Department of Medicine, Section of Cardiology, University of Chicago, 584 I South Maryland Avenue, MC 5084, Chicago, IL 60637, USA

Tel +l 7737021843

Fax + I 7738343274

Email amitpatel@uchicago.edu 
used for the diagnosis of CAD. In recent times, positron emission tomography (PET), stress CMR, and CCT have emerged as additional diagnostic techniques. This is evidenced by American and European guidelines prescribing a noninvasive ischemic assessment of patients with at least an intermediate pretest probability of CAD. ${ }^{5,10}$

This review focuses on the evaluation of patients with suspected CAD using noninvasive imaging modalities. Specifically, the diagnostic utility of MPI with SPECT, PET, MCE, CMR, and CCT is explored (Table 1).

\section{Myocardial perfusion}

Coronary blood flow is a well-regulated physiologic phenomenon. In normal epicardial coronary arteries, nitric oxide released by endothelium results in arterial vasodilation. As atherosclerotic disease progresses, endothelial dysfunction occurs. Subsequently, nitric oxide production decreases and is replaced by acetylcholine causing arterial vasoconstriction. ${ }^{11,12}$

Coronary blood flow is autoregulated and remains stable across a widespread range of perfusion pressures. With lower coronary pressures (as is expected distal to a coronary stenosis), the coronary microcirculation vasodilates to ensure preserved coronary blood flow. Conversely, as coronary pressure increases, the microcirculation vasoconstricts. In patients with flow-limiting $\mathrm{CAD}$, these autoregulatory mechanisms lose further capacity to vasodilate during stress. This results in abnormalities in myocardial perfusion, which can be imaged quantitatively and semiquantitatively through a variety of modalities, each with its strengths and limitations. ${ }^{12}$

The fundamental thesis of stress MPI is the differential myocardial perfusion (blood flow) noted between during resting and stress conditions. Regions of myocardium that demonstrate reduced perfusion during stress conditions when compared to rest are suggestive of ischemia. Matched perfusion defects noted during both rest and stress are thought to represent infarcted segments of myocardium (Figure 1).

The choice of tracer used in MPI depends on the imaging modality selected. Radiotracers are used for SPECT and PET imaging, while lipid microspheres are employed with MCE. In patients who undergo CMR or CCT, respectively, gadolinium or iodinated contrast agents are utilized. Regardless of imaging modality, passage of the tracer through the coronary arteries and microcirculation after systemic administration is proportional to the fraction of total cardiac output. In patients with flow-limiting CAD, tracer uptake into the myocardium is impaired and subsequently visualized in a manner specific to the imaging technique.

There has been considerable national growth in cardiac stress testing. Between 1993-1995 and 2008-2010, stress testing has grown by $\sim 60 \%$ in a risk-adjusted analysis. Over the same time period, the incidence of stress testing with

Table I A summary of noninvasive modalities used in myocardial perfusion imaging

\begin{tabular}{|c|c|c|c|c|}
\hline Imaging modality & Stress conditions & Common tracers & General mechanism & Notes \\
\hline SPECT & $\begin{array}{l}\text { Exercise, pharmacologic } \\
\text { agents }\end{array}$ & $\begin{array}{l}\text { Technetium-99m and } \\
\text { thallium-20I }\end{array}$ & $\begin{array}{l}\text { Differential distribution of tracer } \\
\text { and detection of gamma radiation }\end{array}$ & $\begin{array}{l}\text { Low spatial, temporal } \\
\text { resolution; longer } \\
\text { acquisition times; and } \\
\text { radiation exposure }\end{array}$ \\
\hline PET & Pharmacologic agents & $\begin{array}{l}\text { Rubidium-82, nitrogen- } 13 \\
\text { ammonia, and oxygen- } 15\end{array}$ & $\begin{array}{l}\text { Differential distribution of tracer } \\
\text { and detection of gamma radiation }\end{array}$ & $\begin{array}{l}\text { Improved spatial, } \\
\text { temporal resolution } \\
\text { compared to SPECT; } \\
\text { intermediate acquisition } \\
\text { times; and radiation } \\
\text { exposure }\end{array}$ \\
\hline MCE & $\begin{array}{l}\text { Exercise, pharmacologic } \\
\text { agents }\end{array}$ & $\begin{array}{l}\text { Microspheres (with varying } \\
\text { shell and gas core) }\end{array}$ & $\begin{array}{l}\text { Ultrasound detection of } \\
\text { microspheres and wall motion } \\
\text { assessment }\end{array}$ & $\begin{array}{l}\text { High spatial, temporal } \\
\text { resolution and short } \\
\text { acquisition times }\end{array}$ \\
\hline CMR & Pharmacologic agents & $\begin{array}{l}\text { Gadolinium-based contrast } \\
\text { agents }\end{array}$ & First-pass perfusion imaging & $\begin{array}{l}\text { High spatial, temporal } \\
\text { resolution; intermediate } \\
\text { acquisition times; and no } \\
\text { radiation exposure }\end{array}$ \\
\hline СCT & Pharmacologic agents & lodinated contrast agents & $\begin{array}{l}\text { First-pass perfusion imaging and } \\
\text { static or dynamic imaging }\end{array}$ & $\begin{array}{l}\text { High spatial, temporal } \\
\text { resolution; intermediate } \\
\text { acquisition times; and } \\
\text { radiation exposure }\end{array}$ \\
\hline
\end{tabular}

Abbreviations: SPECT, single-photon emission computed tomography; PET, positron emission tomography; MCE, myocardial contrast echocardiography; CMR, cardiovascular magnetic resonance; CCT, cardiovascular computerized tomography. 

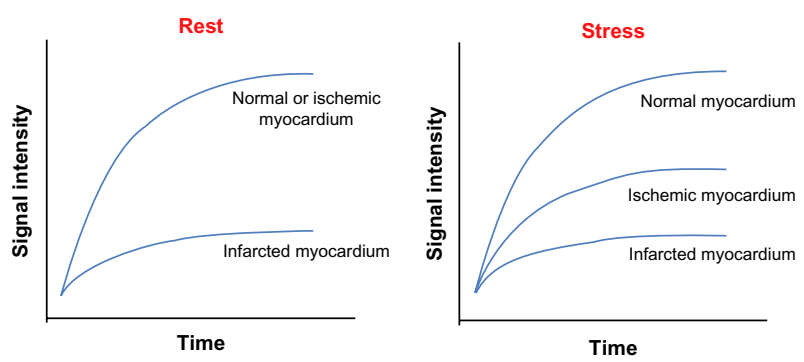

Figure I Representation of myocardial perfusion curves during rest and stress conditions.

imaging has increased from $59 \%$ to $87 \%$. The information provided by MPI has significant diagnostic and prognostic information and is a viable gatekeeper to coronary angiography. ${ }^{13}$

\section{Evaluation of patients with stable CAD}

Patients who are reluctant to undergo invasive coronary angiography can be risk stratified according to a variety of noninvasive modalities utilizing MPI. Ultimately, the ischemic burden identified by noninvasive testing is associated with cardiovascular outcomes.

A large retrospective study of $\sim 11,000$ patients who underwent exercise or pharmacologic SPECT MPI without a prior history of myocardial infarction or previous revascularization was followed for $\sim 2$ years. Based on the SPECT MPI results, it was shown that patients with a lesser burden of ischemic myocardium benefited more with optimal medical management as opposed to revascularization. Only patients with $>10 \%$ of ischemic myocardium benefited from revascularization. This study helped to demonstrate that MPI is useful not only in the diagnosis of CAD but also in risk stratification and in guiding further management. ${ }^{14}$ These findings have been confirmed in a more recent analysis as well. ${ }^{15} \mathrm{An}$ ongoing prospective randomized trial, International Study of Comparative Health Effectiveness with Medical and Invasive Approaches, further evaluating the optimal treatment of patients with stable CAD with a moderate-to-large burden of ischemia are currently underway. ${ }^{16}$

Treatment of patients with stable CAD is largely directed at symptoms. ${ }^{17}$ There is conflicting evidence that percutaneous coronary intervention (PCI) improves survival in patients with stable CAD. A recent, large meta-analysis of $>93,000$ patients with stable CAD evaluated whether revascularization improves prognosis compared to medical treatment. The authors showed improved survival with newer generation of drug-eluting stents when compared to medical therapy. ${ }^{18}$
On the other hand, two additional meta-analyses comprising 7,200 and 8,000 patients found no difference in mortality in patients treated with PCI compared to optimal medical therapy. ${ }^{19,20}$

The Fractional Flow Reserve Versus Angiography for Multivessel Evaluation 2 (FAME 2) trial examined patients with stable CAD for whom PCI was being considered. Approximately 1,200 patients underwent invasive coronary angiography with measurement of fractional flow reserve (FFR) in all coronary stenosis. Patients with FFR $<0.80$ were then randomly assigned to PCI plus medical therapy or medial therapy alone. For the composite primary end point of death, myocardial infarction, or urgent revascularization, FFR-guided PCI plus medical therapy was better than medical therapy alone. When individual outcomes of the composite end point were analyzed, there was no difference in mortality or myocardial infarction; the end point was driven entirely by urgent revascularization. ${ }^{21}$

Evaluating patients with stable angina presents the clinician with a variety of options apart from invasive coronary angiography. Noninvasive testing offers both diagnostic and prognostic information. Additionally, it is not clear that revascularization of patients confers a survival benefit.

\section{Single-photon emission computerized tomography Overview}

The assessment of patients with suspected or known CAD using stress testing with SPECT MPI has undergone considerable evolution in the past three decades. Improvements in protocols, pharmacologic stress agents, tracers, and software/ hardware have simplified acquisition and also enhanced the diagnostic utility of SPECT. While there are notable limitations, SPECT remains one of the most widely accepted noninvasive modalities to assess for ischemia (Figure 2).

For patients reluctant to undergo angiography, a major benefit to stress testing with SPECT MPI is its widespread availability. Additionally, a variety of techniques can be used to induce hyperemic conditions. When a patient is unable to exercise, pharmacologic vasodilators (commonly regadenoson, adenosine, or dipyridamole) or dobutamine are typically used. Although thallium-201 is still used especially in the assessment of viability, the most commonly used radionuclide agent is technetium-99m (either Tc-99m sestamibi or Tc-99m tetrofosmin).

There are a number of benefits and limitations of SPECT MPI. Since imaging is not obtained during first-pass perfusion of contrast, a lower temporal resolution is acceptable. 


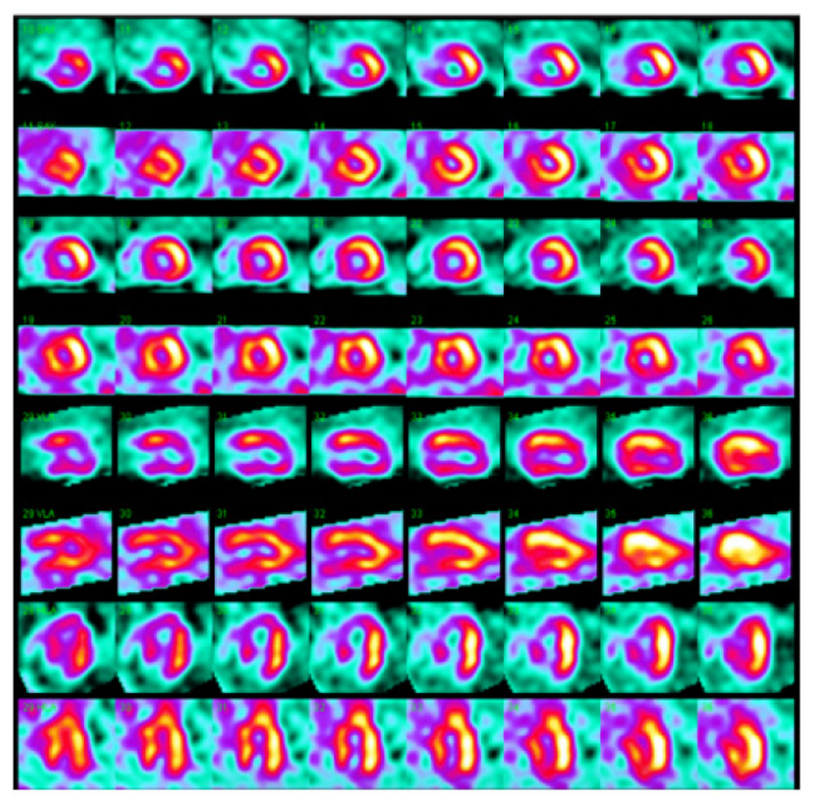

Figure 2 A 76-year-old man with a previous history of myocardial infarction was admitted for angina and subsequently referred for SPECT.

Notes: A large-sized, severe perfusion defect that is predominantly reversible is seen in the left anterior descending artery territory. In addition, a small-sized, moderate perfusion defect is noted in the left circumflex artery territory that is completely reversible and a medium-sized, moderate defect is noted in the right coronary artery territory that is predominantly reversible.

Abbreviation: SPECT, single-photon emission computed tomography.

Consequently, image acquisition is typically acquired over a longer period of time to improve signal-to-noise ratio. The poor spatial resolution compared to newer imaging modalities also mitigates the ability of SPECT MPI to detect subendocardial perfusion defects. ${ }^{22}$ Increasing public health concerns over radiation exposure have also prompted methods to reduce overall radiation doses. ${ }^{23,24}$ In particular, newer hardware (gamma cameras) and software have improved SPECT MPI's diagnostic ability while reducing radiation exposure. $^{25,26}$

\section{Assessment of CAD}

SPECT MPI performs well in the diagnosis of CAD. An earlier pooled analysis of $\sim 9,000$ patients from 79 studies showed an average sensitivity and specificity for the diagnosis of CAD with lesions of $>50 \%$ stenosis of $86 \%$ and $74 \%$, respectively. ${ }^{27}$ Compared to invasive coronary angiography with FFR, a recently published meta-analysis examined the diagnostic accuracy of stress MPI. A total of 924 vessels and 533 patients were examined. The sensitivity and specificity of SPECT MPI for detecting hemodynamically significant CAD was $61 \%$ and $84 \%$ at the vessel level and $74 \%$ and $79 \%$ at the patient level, respectively. ${ }^{28}$ This is similar to what has been observed in prospective studies comparing SPECT to invasive coronary angiography. ${ }^{29}$
Stress testing with SPECT MPI also allows for powerful prognostic information. Patients stratified to low and intermediate risks are often treated medically and observed for changes in symptoms, while high-risk patients are referred for angiography. A large meta-analysis of $\sim 70,000$ patients who underwent SPECT MPI for evaluation of ischemia showed that the annual event rate for low-risk patients was 1.6 versus 14.3 for high-risk patients (a mean follow-up of 2.3 years). ${ }^{30}$ Risk is determined by summed stress score, a semiquantitative index of perfusion abnormalities reflecting infarction or ischemia. When combined with the summed rest score, the difference reflects the ischemic burden. A summed stress score $<4$ is considered as normal to low risk.

Beyond assessment of ischemia in the setting of CAD, SPECT MPI also provides a wealth of information on overall cardiac function and prognosis. While assessment of left ventricular (LV) function necessitates dedicated ventriculogram during angiography (thereby increasing iodinated contrast dose), ECG-gating during SPECT MPI allows for quantification of LV ejection fraction and volume. LV filling pressures also allow for estimation of diastolic function. Additionally, dilation of the LV post stress and uptake of tracer in the right ventricle are negative prognostic features..$^{31,32}$

Importantly, the utility of SPECT MPI in clinical outcomes has been well established. In a substudy of the COURAGE trial, outcomes were analyzed in 1,381 patients with stable CAD on optimal medical therapy randomized to receive PCI or not. Each patient underwent baseline SPECT MPI and was stratified into low- or high-risk categories. The primary end point of death or myocardial infarction was not statistically different with any degree of ischemia observed on baseline SPECT MPI. ${ }^{33}$ These results suggest that patients with CAD can readily be identified with SPECT MPI; subsequent medical management results in similar results in patients treated with revascularization and medical management. While the degree of ischemia on baseline SPECT MPI in this study did not correlate with primary end point, it did identify patients with ischemia and help direct appropriate medical therapy.

\section{Positron emission tomography Overview}

PET has been a mainstay noninvasive imaging modality for the assessment of CAD. In addition, metabolism and ventricular function are obtained from PET imaging. PET imaging improves upon many of the limitations of SPECT. Notably, the ability to quantitate myocardial blood flow (MBF) and acquire images with fewer attenuation artifacts 
allows for more precise identification of abnormal perfusion. Additionally, scanning time is typically shorter with PET $\mathrm{MPI}^{34}$ (Figure 3).

With PET imaging, a radiotracer isotope with a short half-life is injected into the systemic circulation. Most commonly, rubidium-82, nitrogen-13 ammonia, and oxygen-15 are used. Compared to tracers used in SPECT, these tracers track MBF better. Positrons are emitted over time as the radiotracer isotope undergoes beta decay. Released positrons ultimately interact with negatively charged electrons causing the annihilation of both and the generation of photons. A scintillator detects these photons, and subsequently, an image localizing the source is generated.

MPI with PET is performed with stress testing as a means to enhance the ability to detect ischemia in patients with known or suspected CAD.

\section{Assessment of CAD}

PET imaging is often considered the reference standard for the noninvasive evaluation of CAD. A meta-analysis of $\sim 900$ patients from nine studies reported a sensitivity and specificity of $90 \%$ and $89 \%$, respectively, for the detection of CAD with vessel stenosis $>50 \% .{ }^{35}$ Similarly, a more recent analysis showed similar results for the accuracy in detecting CAD when compared to invasive coronary angiography with FFR; the sensitivity and specificity were $83 \%$ and $89 \%$ at the vessel level and $88 \%$ and $80 \%$ at the patient level, respectively. ${ }^{28}$

Owing to the short half-life of radiotracers, PET MPI often requires pharmacologic-induced hyperemia. A recent study, however, demonstrated that exercise testing is feasible. In a study of 265 obese patients who underwent PET MPI, stress testing with treadmill exercise yielded reasonable diagnostic accuracy for CAD. ${ }^{36}$

PET MPI is also valuable for predicting future clinical events. A normal PET MPI scan confers a low annual risk

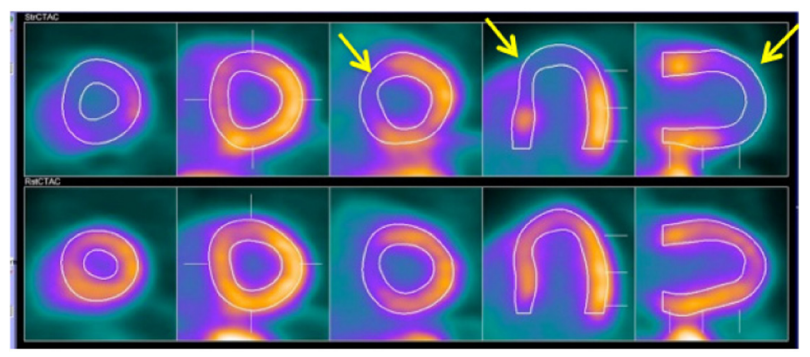

Figure 3 An example of adenosine stress testing with rubidium-82 PET in a patient with suspected CAD.

Notes: A sizeable perfusion defect suggestive of reversible ischemia is noted in the left anterior descending artery territory. The arrows indicate the perfusion defect. Abbreviations: PET, positron emission tomography; CAD, coronary artery disease.
$(<1 \%)$ for cardiac events, whereas patients with an abnormal PET MPI have a much higher annual event rate ranging from $5 \%$ to $10 \%$ in a composite of studies. ${ }^{37,38}$

Importantly, compared to other noninvasive imaging modalities, PET offers the advantage of allowing for absolute MBF. This has been shown to aid in the real-world diagnosis of CAD in addition to prognostication. ${ }^{39-42}$ In one study of 2,783 patients referred for rest/stress PET for assessment of $\mathrm{CAD}$, quantification of MBF conferred incremental risk stratification over semiquantitative assessment of ischemia. ${ }^{43}$

\section{Myocardial contrast echocardiography Overview}

In addition to better delineate endocardial boundaries and opacification of the LV, echocardiographic contrast agents are an effective intravascular tracer utilized for interrogation of myocardial perfusion. As such, MCE represents a wellvalidated imaging modality for the assessment of CAD.

Lipid microspheres have emerged overtime as a reliable echocardiographic contrast agent. Microspheres vary according to their outer shell composition (providing differing hydrophilic properties) and gas content (conferring differing echogenic properties). After injection of microspheres into the systemic circulation, the ultrasound-induced oscillation reflects a unique echo allowing for differentiation of blood, myocardium, and other tissues. ${ }^{44}$

MBF using MCE was first described in dog models $>20$ years ago. ${ }^{45}$ After opacification of the LV cavity with contrast, a high-intensity ultrasound pulse causes destruction of the microspheres. In normal myocardium, replenishment of the LV with contrast takes $\sim 5$ seconds. Under hyperemic conditions causing vasodilation of the arteriolar beds, contrast replenishment typically takes $<2$ seconds. Conversely, decreased MBF due to coronary stenosis results in an increased replenishment time. Quantification of the MBF at stress divided by the MBF at rest results in an assessment of myocardial flow reserve, a surrogate for coronary flow reserve. ${ }^{44,46,47}$ Coronary flow reserve is clinically useful in the evaluation of patients with microcirculatory disease and has been shown to discriminate ischemic from nonischemic cardiomyopathy (sensitivity $91 \%$, specificity $89 \%$ ). ${ }^{48}$

\section{Assessment of CAD}

More commonly, stress echocardiography (with exercise or a pharmacologic agent) and evaluation of ventricular wall motion and geometry are used to detect hemodynamically significant CAD. When contrast is used during stress 
echocardiography, enhanced detection of ischemic changes is possible.

MPI with contrast enhancement is well suited to diagnose CAD. In an analysis of 588 patients from nine trials, there was reasonable concordance between MCE and SPECT (kappa 0.81 at the patient level and 0.86 at the vessel level $)^{49}$ for the diagnosis of CAD. Additionally, when compared to the gold standard of invasive coronary angiography, a meta-analysis of $\sim 1,700$ patients from 20 trials showed a sensitivity of $83 \%$ and a specificity of $80 \%$ for the diagnosis of $\mathrm{CAD}^{50}$ (Figure 4 ).

Furthermore, perfusion imaging with echocardiography provides important prognostic data. The addition of MPI to wall motion analysis added incremental value in a follow-up of 788 patients with known or suspected CAD. Abnormal myocardial perfusion was a better predictor than other clinical factors, ejection fraction, and wall motion. The 3-year event free survival in patients with normal wall motion and myocardial perfusion was $95 \%$ compared to $68 \%$ in patients with abnormal wall motion and myocardial perfusion. ${ }^{51}$ Similarly, another study of 202 patients with known or suspected CAD who underwent stress echocardiography with MPI showed a hazard ratio of 6.1 for major adverse cardiovascular events in patients with any inducible perfusion defect or wall motion abnormality (a mean follow-up of 32 months). ${ }^{52}$

In patients with acute coronary syndrome, MCE has also proven to be a valuable tool. In $>500$ patients presenting with ACS, normal troponin levels, and a nondiagnostic ECG, the presence of abnormal wall motion and myocardial perfusion yielded a hazard ratio of 10.7 for prediction of future cardiac events. ${ }^{53}$

MCE has several notable advantages when compared to SPECT. Echocardiography is typically widely available
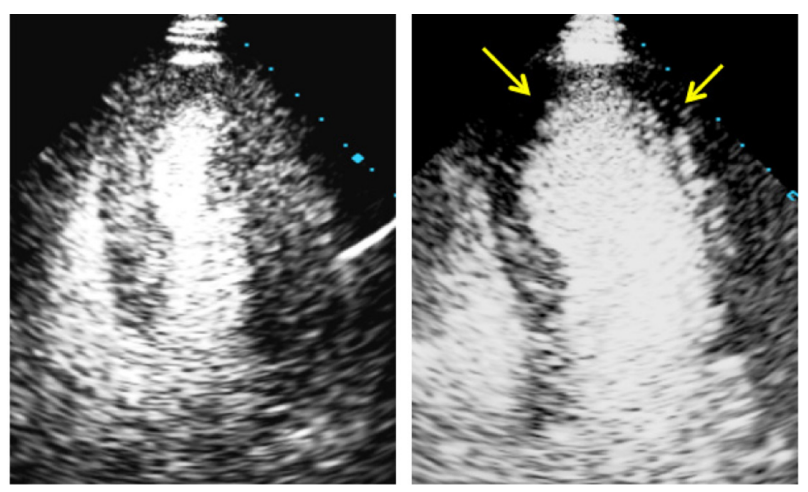

Figure 4 An example of MCE imaging showing normal enhancement at rest (left) and delayed replenishment with adenosine (resulting in low-contrast enhancement in midseptal, apical septal, and apical lateral segments) consistent with stenosis in the left anterior descending artery (right).

Note: The arrows represent abnormal perfusion with contrast.

Abbreviation: MCE, myocardial contrast echocardiography. even compared to SPECT. Improved spatial resolution of MCE allows for better discrimination of subendocardial perfusion defects that otherwise would be missed by SPECT. Additionally, the lack of ionizing radiation and quicker image acquisition time are both favorably viewed by patients. Also, unlike SPECT, MCE allows for absolute quantification of MBF, which conceivably could be followed over time in high-risk patients. The challenge with MCE, however, is obtaining adequate acoustic windows, often limited by body habitus. The lower signal-to-noise ratio often makes images challenging to interpret.

Perfusion imaging with echocardiography is a reliable and important tool for the diagnosis of CAD. In patients hesitant to the idea of invasive angiography, MCE is a wellvalidated noninvasive imaging modality for both diagnosis and prognosis of CAD.

\section{Cardiovascular magnetic resonance Overview}

During the last decade, CMR has emerged as an excellent imaging modality useful for the quantitative and qualitative assessments of CAD via perfusion imaging. Beyond functional assessment of the patient, CMR also provides a plethora of anatomic and structural details useful to the clinician. With wider acceptance and availability of CMR, it is expected that this noninvasive imaging modality will continue to play an increasingly important role in the evaluation of patients with cardiovascular disease.

MPI using CMR is usually performed during a stress study. While a variety of protocols exist, stress perfusion imaging is normally done after administration of a pharmacologic vasodilator and a gadolinium-based contrast agent. Typically, stress images are acquired first followed by recovery images once hyperemic conditions start to return to baseline. ${ }^{54} \mathrm{MPI}$ is achieved during first pass of contrast using a variety of T1-weighted pulse sequences depending on the equipment vendor and level of expertise of the physician. Late gadolinium enhancement is subsequently performed in multiple planes using an inversion recovery technique. At experienced centers, the study usually takes $30-60$ minutes. ${ }^{22}$

Myocardial perfusion can be assessed qualitatively, semiquantitatively, or quantitatively. The simplest semiquantitative approach includes adjudicating the burden of perfusion defects visually and estimating the ischemic fraction in territories of myocardium supplied by an epicardial coronary artery. Additionally, more advanced semiquantitative analysis can be performed using myocardial perfusion reserve index. The myocardial time-intensity curves of contrast entering 
the LV cavity generated from first-pass perfusion allow for measurement of the slope of the signal-intensity curve during early contrast enhancement of the LV cavity and also the myocardium. The ratio of these slopes at stress and rest represents the myocardial perfusion reserve index and is a surrogate marker for the coronary microcirculation. ${ }^{55,56}$ Fully quantitative methods to calculate myocardial perfusion allow for the measurement of absolute MBF. While generally more time intensive, quantitative approaches have higher diagnostic accuracy for detecting hemodynamically significant $\mathrm{CAD}^{57}$ and may better differentiate patients with single vessel coronary disease from those with triple vessel CAD. ${ }^{58}$

As CMR continues to evolve, the advent of blood oxygenation level-dependent (BOLD) imaging is an emerging perfusion technique that holds much promise. BOLD imaging requires no exogenous contrast and instead takes advantage of the differences in T2 signal between oxygenated and deoxygenated blood. As such, differences in myocardial perfusion differentiating between normal and ischemic myocardium are quantifiable. BOLD imaging has been demonstrated to detect ischemia in stress and rest in preclinical models and also in early human trials..$^{59,60}$

Stress CMR confers several advantages over other noninvasive imaging modalities. The high spatial and temporal resolutions of CMR in addition to lack of ionizing radiation allow for better delineation of anatomic, structural, and functional information. When compared to SPECT, CMR has improved ability to detect subendocardial perfusion defects. ${ }^{22}$

A limitation of stress CMR is that typically only three short-axis slices of the left ventricle can be imaged during the stress perfusion acquisition; however, as image acquisition techniques have become quicker, complete LV coverage is now feasible. ${ }^{61,62}$ Similarly, nearly automated techniques to quantify myocardial perfusion have been developed to allow for quicker analysis than the time consuming and cumbersome manual methods currently being used to quantify myocardial perfusion. ${ }^{63}$ In addition, while generally considered safe, there is some controversy that the magnetic and radiofrequency field generated during CMR may cause blood cell alterations and DNA damage in the short term; however, this finding has not been consistently replicated and its clinical relevance is unknown. ${ }^{64,65}$ Unfortunately, certain patients are generally excluded from undergoing stress CMR. These may include patients with significant claustrophobia, patients unable to cooperate with breath holds, patients with advanced renal disease who are at risk for nephrogenic systemic fibrosis, and patients with some implanted metal devices (such as certain pacemakers or defibrillators; ${ }^{22}$ Figures 5 and 6).

\section{Assessment of CAD}

The excellent diagnostic and prognostic utility of CMR for the diagnosis of CAD has been established in recent years. Analysis of MR-IMPACT and MR-IMPACT II, two multi-center studies that compared stress CMR to SPECT in the assessment of ischemia showed that the sensitivity of stress CMR is greater than SPECT for the diagnosis of CAD. ${ }^{66,67}$

The largest prospective trial to date also showed similar results. In the CE-MARC study, 628 patients with suspected CAD underwent stress CMR, SPECT, and invasive coronary angiography. The sensitivity, specificity, positive predictive value, and negative predictive value to detect clinical significant CAD (defined as $>70 \%$ stenosis of a coronary artery or $>50 \%$ left main stem stenosis) was $86.5 \%, 83.4 \%, 77.2 \%$, and $90.5 \%$, respectively, for CMR and $66.5 \%, 82.5 \%, 71.4 \%$, and $79.1 \%$, respectively, for SPECT. ${ }^{29}$
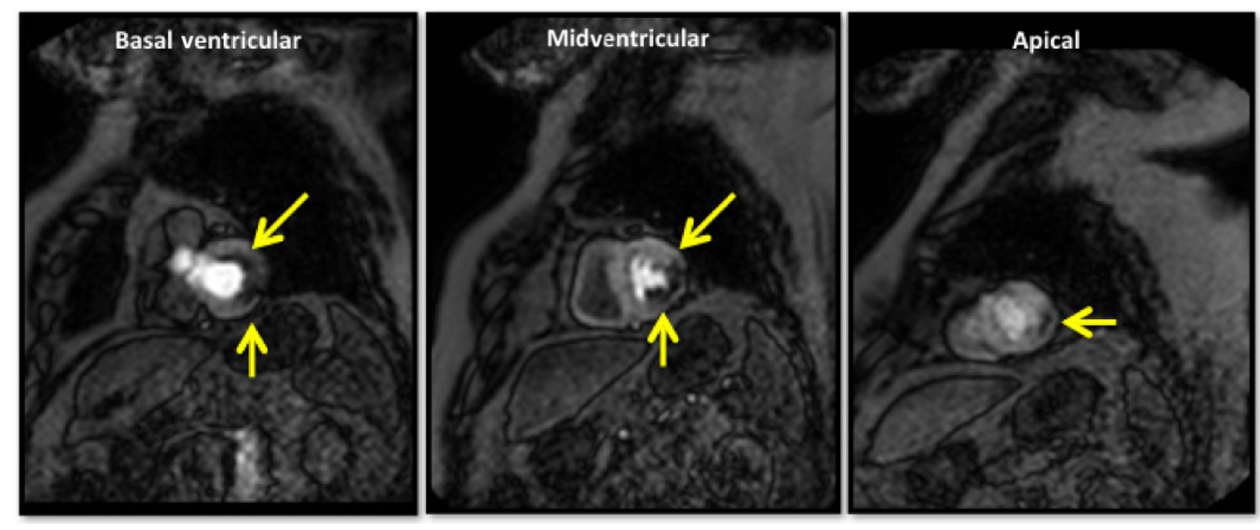

Figure 5 A 77-year-old man with previous coronary artery bypass grafting and worsening dyspnea with exertion presented for stress CMR. Note: A large, severe perfusion defect was present in the basal/mid inferolateral and anterolateral walls and apical lateral wall (yellow arrows). Abbreviation: CMR, cardiovascular magnetic resonance. 

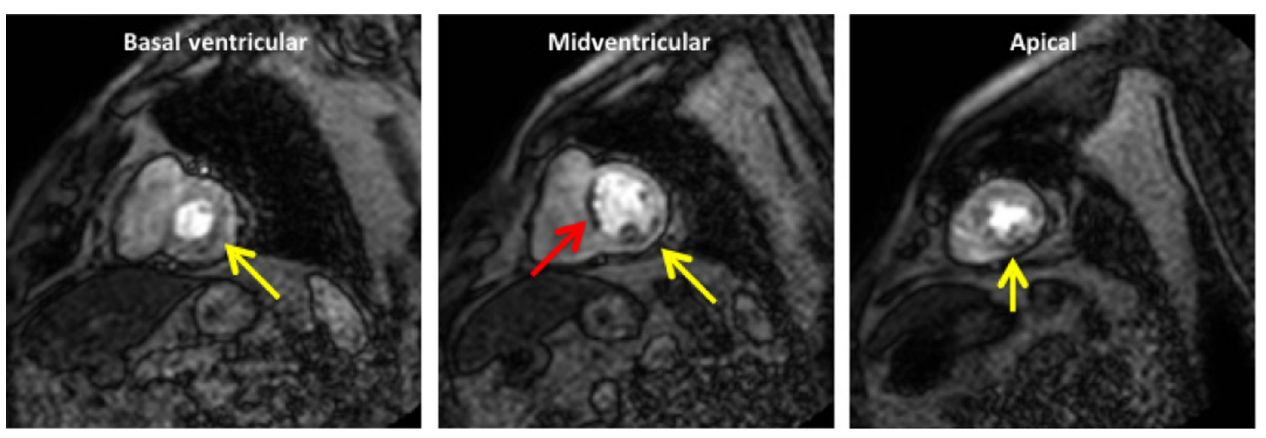

Figure 6 An 83-year-old man with previous history of multivessel coronary revascularization presented with recurrence of his angina.

Notes: Stress CMR demonstrated the presence of two perfusion defects. The first (yellow arrows) was a mild-moderate defect in the basal and mid inferolateral walls and apical inferior wall. The second (red arrow) was a severe perfusion defect in the mid anteroseptum.

Abbreviation: CMR, cardiovascular magnetic resonance.

When using invasive coronary angiography with FFR as the gold standard to adjudicate flow-limiting CAD, a recent meta-analysis showed a sensitivity and specificity of $87 \%$ and $91 \%$ for diagnosis of CAD the vessel level and $89 \%$ and $87 \%$ at the patient level. CMR outperformed all other non-invasive imaging modalities including SPECT, CCT, and MCE. ${ }^{28}$

The prognostic information derived from stress CMR is important in assessing patients unwilling to undergo invasive coronary angiography. One study reported a negative predictive value of $99 \%$ for major adverse cardiovascular events in patients with known or suspected CAD without perfusion defects or other abnormalities noted on stress CMR ${ }^{68}$ In addition, another study demonstrated abnormalities at various points of the ischemic cascade (no reversible ischemia, stress perfusion defect alone, and stress perfusion defect with wall motion abnormality) identified by stress CMR correlates with adverse cardiac events in the long-term follow-up. ${ }^{69}$

\section{Cardiovascular computerized tomography}

The role of CCT coronary angiography has been well established in evaluating patients with symptoms suggestive of CAD by characterizing the presence, location, and severity of a coronary stenosis. However, CCT angiography is often limited by blooming artifacts due to coronary calcification that prevents assessment of the coronary lumen and in its ability to differentiate flow-limiting from nonhemodynamically significant lesions. Coupling CCT angiography with myocardial perfusion assessment is a promising technique that allows for the assessment of the physiologic significance of a coronary stenosis in addition to its anatomic appearance. ${ }^{70}$

Analogous to CMR, the assessment of myocardial perfusion with CCT is based on first pass of iodinated contrast through the myocardium. Ischemic territories of myocardium are identified by hypoattenuation of signal intensity. The use of vasodilators further allows for delineation between perfusion abnormalities between resting and stress states (Figure 7).

Static and dynamic CT imaging techniques are the primary techniques utilized for the evaluation of myocardial perfusion. Static imaging is acquired during the initial phase of contrast injection (at peak contrast concentration) and allows for assessment of the myocardium at a single point in time. Images are typically acquired during resting conditions at the same time as CCT coronary angiography and separately during hyperemia. Static myocardial perfusion is often interpreted visually with attenuation of signal seen in ischemic myocardium. The sensitivity and specificity of diagnosing significant $\mathrm{CAD}$ with static imaging have ranged $50 \%-96 \%$ and $68 \%-98 \%$, respectively, among multiple studies. ${ }^{71}$ In patients with flow-limiting CAD demonstrated by FFR, CCT MPI (with static imaging) was shown to be accurate in identifying ischemic vessels and was better than CCT angiography alone. ${ }^{72}$

Dynamic CT imaging allows for a more comprehensive measurement of myocardial perfusion. Unlike static imaging where a single frame is acquired, dynamic imaging technique acquires multiple frames during first-pass contrast uptake, similar to stress CMR and PET. As such, both semiquantitative and quantitative approaches to assessing myocardial perfusion are possible. Given the novelty of this technique, only studies with small numbers of patients have been done. The overall sensitivity and specificity are improved compared to static imaging and range between $76 \%$ and $100 \%$ and $74 \%$ and $100 \%$, respectively. ${ }^{71}$

Abnormal myocardial perfusion can be detected with resting or vasodilator CCT MPI. Rest CCT MPI has reliably been shown to detect perfusion defects confirmed 

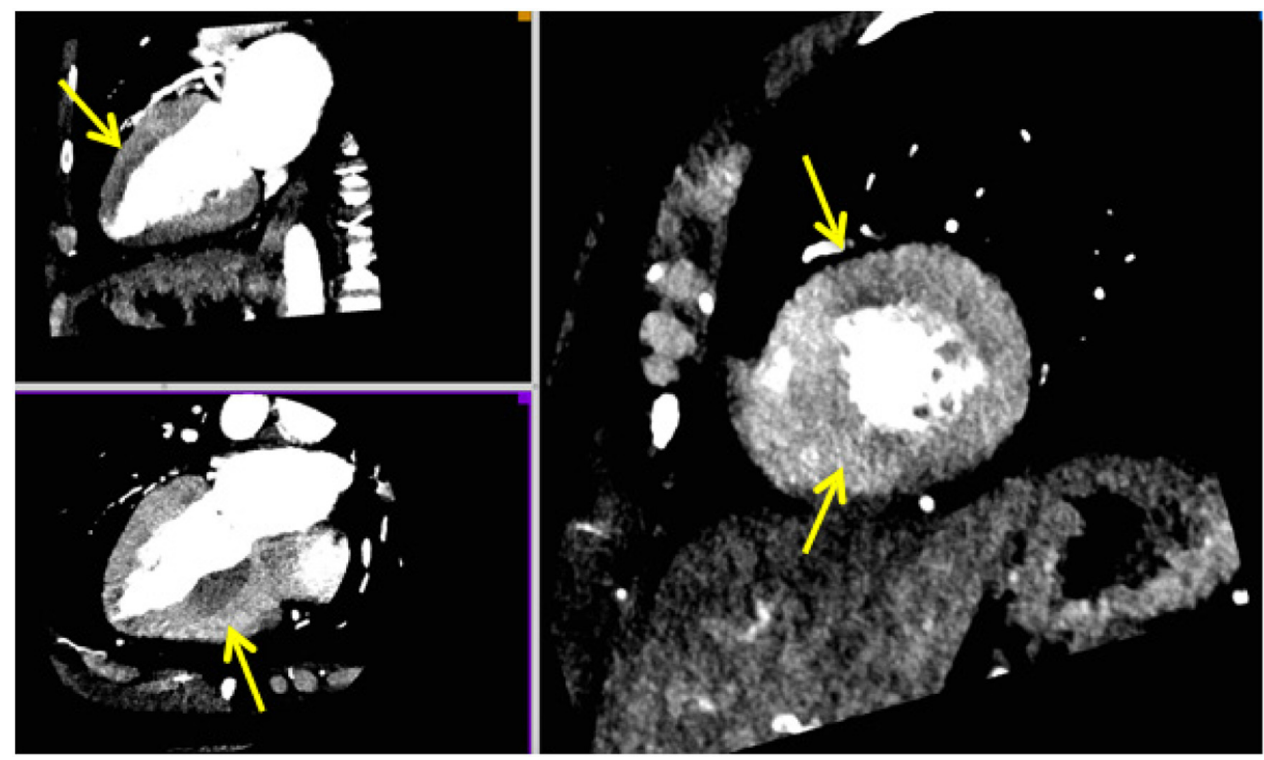

Figure 7 An example of perfusion defects (yellow arrows) seen in the anterior wall and inferoseptum using CCT in the long axis (left) and short axis (right). Abbreviation: CCT, cardiovascular computerized tomography.

with SPECT MPI or CMR. ${ }^{73-75}$ The improved spatial resolution of CCT MPI over SPECT allows for improved identification of subtle perfusion defects. Moreover, rest CCT MPI requires no further contrast administration or radiation exposure. Vasodilator CCT MPI allows for a more comprehensive diagnostic capability. The multicenter CORE320 study evaluated a combined approach of CCT angiography and CCT MPI in $~ 400$ patients. The authors found that the receiving operator characteristic curve for combined technique for detecting or excluding flow-limiting lesions was $0.87 .{ }^{76}$ Novel approaches such as qualitative three-dimensional evaluation of myocardial perfusion and iterative reconstruction using vasodilator CCT MPI also improve the detection of CAD. ${ }^{77,78}$

\section{Other considerations}

The presence of myocardial ischemia without overt manifestations of angina or its equivalent is known as silent ischemia. The noninvasive evaluation of silent ischemia is controversial. ${ }^{79}$ Nonetheless, the presence of silent ischemia carries a worse prognosis. Concerns about silent ischemia have been evaluated with MPI. One study of 592 patients who underwent MPI with SPECT or PET showed that the prevalence of ischemia detected by MPI was similar in patients with or without symptoms. ${ }^{80}$ Furthermore, another study examined the value of SPECT MPI in patients 5 years post PCI. This study found similar rates of major adverse cardiovascular events in patients with symptomatic or silent ischemia. Additionally, the authors noted that abnormal MPI is most useful when progression of CAD is noted in remote territories from the area of previous intervention. ${ }^{81}$ No consensus exists on the treatment of patients with silent ischemia, and further trials are needed to assess the role of MPI in its detection and impact on outcomes.

The ability to identify and differentiate between stable and vulnerable coronary plaque using a noninvasive approach could aid in further risk stratification and management. This is an active area of investigation, as plaque characteristics may correlate with hemodynamically significant flow limitations (similar to FFR) more so than the degree of stenosis noted in coronary angiography. Notably, recent works using CCT have demonstrated success in identifying hemodynamically significant CAD through plaque characterization. ${ }^{82,83}$ Similarly, recent works using PET or SPECT to identify vulnerable plaques may further support the role for noninvasive imaging in identifying higher risk patients. ${ }^{84,85}$

\section{Appropriate use of noninvasive imaging}

Despite the plethora of options available to the clinician to evaluate patients with suspected CAD, precautions should be taken to ensure that the correct ischemic testing is ordered. In addition, given the widespread movements to safeguard financial resources, all testing should fall within the purview of appropriateness. American and European guidelines advocated for noninvasive testing in patients with an intermediate pretest probability for CAD. ${ }^{5,10}$ Furthermore, specific guidelines outlining appropriate use in patients with stable 
ischemic heart disease help clinicians navigate the complex variety of options available for testing. ${ }^{86}$

\section{Conclusion}

The increasing burden of CAD presents clinicians with a variety of management decisions and challenges. The ultimate aim should be directed toward relieving symptoms of angina and improving survival. In patients with stable CAD, revascularization does not convincingly improve survival over optimal medical therapy. In patients hesitant to undergo invasive coronary angiography, it is reassuring that a variety of noninvasive imaging modalities exist that provide high-quality diagnostic and prognostic information. The choice of imaging modality is often dependent on availability, clinician preference and comfort level, and patient characteristics.

\section{Acknowledgment}

This study received research support from Astellas and Philips.

\section{Disclosure}

The authors report no conflicts of interest in this work.

\section{References}

1. Nabel EG, Braunwald E. A tale of coronary artery disease and myocardial infarction. N Engl J Med. 2012;366:54-63.

2. Mozaffarian D, Benjamin EJ, Go AS, et al. Heart disease and stroke statistics - 2015 update: a report from the American Heart Association. Circulation. 2015;131:e29-e322.

3. Gordon T, Kannel WB, Hjortland MC, McNamara PM. Menopause and coronary heart disease. The Framingham study. Ann Intern Med. 1978;89:157-161.

4. Lerner DJ, Kannel WB. Patterns of coronary heart disease morbidity and mortality in the sexes: a 26-year follow-up of the Framingham population. Am Heart J. 1986;111:383-390.

5. Fihn SD, Gardin JM, Abrams J, et al; American College of Cardiology Foundation; American Heart Association Task Force on Practice Guidelines; American College of Physicians; American Association for Thoracic Surgery; Preventive Cardiovascular Nurses Association; Society for Cardiovascular Angiography and Interventions; Society of Thoracic Surgeons. 2012 ACCF/AHA/ACP/AATS/PCNA/SCAI/STS Guideline for the diagnosis and management of patients with stable ischemic heart disease: a report of the American College of Cardiology Foundation/ American Heart Association Task Force on Practice Guidelines, and the American College of Physicians, American Association for Thoracic Surgery, Preventive Cardiovascular Nurses Association, Society for Cardiovascular Angiography and Interventions, and Society of Thoracic Surgeons. J Am Coll Cardiol. 2012;60:e44-e164.

6. Halpern EJ, Fischman D, Savage MP, Koka AR, DeCaro M, Levin DC. Decision analytic model for evaluation of suspected coronary disease with stress testing and coronary CT angiography. Acad Radiol. 2010; 17:577-586.

7. Patel MR, Peterson ED, Dai D, et al. Low diagnostic yield of elective coronary angiography. $N$ Engl J Med. 2010;362:886-895.

8. Ammann P, Brunner-La Rocca HP, Angehrn W, Roelli H, Sagmeister M, Rickli H. Procedural complications following diagnostic coronary angiography are related to the operator's experience and the catheter size. Catheter Cardiovasc Interv. 2003;59:13-18.
9. Schönenberger E, Schnapauff D, Teige F, Laule M, Hamm B, Dewey M. Patient acceptance of noninvasive and invasive coronary angiography. PLoS One. 2007;2:e246.

10. Task Force Members, Montalescot G, Sechtem U, et al; ESC Committee for Practice Guidelines; Document Reviewers. 2013 ESC guidelines on the management of stable coronary artery disease: the task force on the management of stable coronary artery disease of the European Society of Cardiology. Eur Heart J. 2013;34:2949-3003.

11. Zeiher AM, Drexler H, Wollschläger H, Just H. Endothelial dysfunction of the coronary microvasculature is associated with coronary blood flow regulation in patients with early atherosclerosis. Circulation. 1991;84:1984-1992.

12. Duncker DJ, Koller A, Merkus D, Canty JM. Regulation of coronary blood flow in health and ischemic heart disease. Prog Cardiovasc Dis. 2015;57:409-422.

13. Ladapo JA, Blecker S, Douglas PS. Physician decision making and trends in the use of cardiac stress testing in the United States: an analysis of repeated cross-sectional data. Ann Intern Med. 2014;161:482-490.

14. Hachamovitch R, Hayes SW, Friedman JD, Cohen I, Berman DS. Comparison of the short-term survival benefit associated with revascularization compared with medical therapy in patients with no prior coronary artery disease undergoing stress myocardial perfusion single photon emission computed tomography. Circulation. 2003;107:2900-2907.

15. Nudi F, Neri G, Schillaci O, et al. Time to and risk of cardiac events after myocardial perfusion scintigraphy. J Cardiol. 2015;66:125-129.

16. New York University School of Medicine. International Study of Comparative Health Effectiveness With Medical and Invasive Approaches (ISCHEMIA) (ISCHEMIA). Available from: https://clinicaltrials.gov/ ct2/show/NCT01471522. NLM identifier: NCT01471522. Accessed April 11, 2016.

17. Boden WE, O'Rourke RA, Teo KK, et al. Optimal medical therapy with or without PCI for stable coronary disease. $N$ Engl J Med. 2007;356:1503-1516.

18. Windecker S, Stortecky S, Stefanini GG, et al. Revascularisation versus medical treatment in patients with stable coronary artery disease: network meta-analysis. BMJ. 2014;348:g3859.

19. Stergiopoulos K, Brown DL. Initial coronary stent implantation with medical therapy vs medical therapy alone for stable coronary artery disease: meta-analysis of randomized controlled trials. Arch Intern Med. 2012;172:312-319.

20. Bangalore S, Pursnani S, Kumar S, Bagos PG. Percutaneous coronary intervention versus optimal medical therapy for prevention of spontaneous myocardial infarction in subjects with stable ischemic heart disease. Circulation. 2013;127:769-781.

21. De Bruyne B, Pijls NH, Kalesan B, et al. Fractional flow reserve-guided pci versus medical therapy in stable coronary disease. $N$ Engl $J$ Med. 2012;367:991-1001.

22. Salerno M, Beller GA. Noninvasive assessment of myocardial perfusion. Circ Cardiovasc Imaging. 2009;2:412-424.

23. Fazel R, Shaw LJ. Radiation exposure from radionuclide myocardial perfusion imaging: concerns and solutions. J Nucl Cardiol. 2011;18:562-565.

24. Einstein AJ, Pascual TN, Mercuri M, et al. Current worldwide nuclear cardiology practices and radiation exposure: results from the 65 country IAEA Nuclear Cardiology Protocols Cross-Sectional Study (INCAPS). Eur Heart J. 2015;36:1689-1696.

25. Palyo R, Sinusas A, Liu YH. High-sensitivity and high-resolution SPECT/CT systems provide substantial dose reduction without compromising quantitative precision for assessment of myocardial perfusion or function. J Nucl Med. Epub 2016 Feb 4.

26. Piccinelli M, Garcia EV. Advances in single-photon emission computed tomography hardware and software. Cardiol Clin. 2016;34:1-11.

27. Underwood SR, Anagnostopoulos C, Cerqueira M, et al; British Cardiac Society; British Nuclear Cardiology Society; British Nuclear Medicine Society; Royal College of Physicians of London; Royal College of Radiologists. Myocardial perfusion scintigraphy: the evidence. Eur $J$ Nucl Med Mol Imaging. 2004;31:261-291. 
28. Takx RA, Blomberg BA, El Aidi H, et al. Diagnostic accuracy of stress myocardial perfusion imaging compared to invasive coronary angiography with fractional flow reserve meta-analysis. Circ Cardiovasc Imaging. 2015;8:e002666.

29. Greenwood JP, Maredia N, Younger JF, et al. Cardiovascular magnetic resonance and single-photon emission computed tomography for diagnosis of coronary heart disease (CE-MARC): a prospective trial. Lancet. 2012;379:453-460.

30. Shaw LJ, Iskandrian AE. Prognostic value of gated myocardial perfusion SPECT. J Nucl Cardiol. 2004;11:171-185.

31. Abidov A, Germano G, Berman DS. Transient ischemic dilation ratio: a universal high-risk diagnostic marker in myocardial perfusion imaging. J Nucl Cardiol. 2007;14:497-500.

32. Mannting F, Zabrodina YV, Dass C. Significance of increased right ventricular uptake on $99 \mathrm{mtc}$-sestamibi SPECT in patients with coronary artery disease. J Nucl Med. 1999;40:889-894.

33. Shaw LJ, Weintraub WS, Maron DJ, et al. Baseline stress myocardial perfusion imaging results and outcomes in patients with stable ischemic heart disease randomized to optimal medical therapy with or without percutaneous coronary intervention. Am Heart J. 2012;164:243-250.

34. Ghotbi AA, Kjaer A, Hasbak P. Review: comparison of PET rubidium-82 with conventional SPECT myocardial perfusion imaging. Clin Physiol Funct Imaging. 2014;34:163-170.

35. Di Carli MF, Hachamovitch R. New technology for noninvasive evaluation of coronary artery disease. Circulation. 2007;115:1464-1480.

36. Aggarwal NR, Drozdova A, Wells Askew J, Kemp BJ, Chareonthaitawee P. Feasibility and diagnostic accuracy of exercise treadmill nitrogen-13 ammonia PET myocardial perfusion imaging of obese patients. $\mathrm{J} \mathrm{Nucl}$ Cardiol. 2015;22(6):1273-1280.

37. Taqueti VR, Di Carli MF. Radionuclide myocardial perfusion imaging for the evaluation of patients with known or suspected coronary artery disease in the era of multimodality cardiovascular imaging. Prog Cardiovasc Dis. 2015;57:644-653.

38. Dorbala S, Di Carli MF. Cardiac PET perfusion: prognosis, risk stratification, and clinical management. Semin Nucl Med. 2014;44:344-357.

39. Waller AH, Blankstein R, Kwong RY, Di Carli MF. Myocardial blood flow quantification for evaluation of coronary artery disease by positron emission tomography, cardiac magnetic resonance imaging, and computed tomography. Curr Cardiol Rep. 2014;16:483.

40. Schindler TH. Myocardial blood flow: Putting it into clinical perspective. J Nucl Cardiol. Epub 2015 Dec 28.

41. Hagemann CE, Ghotbi AA, Kjær A, Hasbak P. Quantitative myocardial blood flow with rubidium-82 PET: a clinical perspective. Am J Nucl Med Mol Imaging. 2015;5:457-468.

42. Ohira H, Dowsley T, Dwivedi G, et al. Quantification of myocardial blood flow using PET to improve the management of patients with stable ischemic coronary artery disease. Future Cardiol. 2014;10:611-631.

43. Murthy VL, Naya M, Foster CR, et al. Improved cardiac risk assessment with noninvasive measures of coronary flow reserve. Circulation. 2011;124:2215-2224.

44. Pathan F, Marwick TH. Myocardial perfusion imaging using contrast echocardiography. Prog Cardiovasc Dis. 2015;57:632-643.

45. Jayaweera AR, Edwards N, Glasheen WP, Villanueva FS, Abbott RD, Kaul S. In vivo myocardial kinetics of air-filled albumin microbubbles during myocardial contrast echocardiography. Comparison with radiolabeled red blood cells. Circ Res. 1994;74:1157-1165.

46. Vogel R, Indermühle A, Reinhardt J, et al. The quantification of absolute myocardial perfusion in humans by contrast echocardiography: algorithm and validation. J Am Coll Cardiol. 2005;45:754-762.

47. Wei K, Ragosta M, Thorpe J, Coggins M, Moos S, Kaul S. Noninvasive quantification of coronary blood flow reserve in humans using myocardial contrast echocardiography. Circulation. 2001;103: 2560-2565.

48. Senior R, Janardhanan R, Jeetley P, Burden L. Myocardial contrast echocardiography for distinguishing ischemic from nonischemic firstonset acute heart failure: insights into the mechanism of acute heart failure. Circulation. 2005;112:1587-1593.
49. Bhatia VK, Senior R. Contrast echocardiography: evidence for clinical use. J Am Soc Echocardiogr. 2008;21:409-416.

50. Dijkmans PA, Senior R, Becher H, et al. Myocardial contrast echocardiography evolving as a clinically feasible technique for accurate, rapid, and safe assessment of myocardial perfusion: the evidence so far. J Am Coll Cardiol. 2006;48:2168-2177.

51. Tsutsui JM, Mukherjee S, Elhendy A, et al. Value of dobutamine stress myocardial contrast perfusion echocardiography in patients with advanced liver disease. Liver Transpl. 2006;12:592-599.

52. Wejner-Mik P, Lipiec P, Kasprzak JD. Long-term prognostic value of dipyridamole stress myocardial contrast echocardiography. Eur $J$ Echocardiogr. 2011;12:762-766.

53. Gaibazzi N, Squeri A, Reverberi C, et al. Contrast stress-echocardiography predicts cardiac events in patients with suspected acute coronary syndrome but nondiagnostic electrocardiogram and normal 12-hour troponin. J Am Soc Echocardiogr. 2011;24:1333-1341.

54. Bhave NM, Freed BH, Yodwut $\mathrm{C}$, et al. Considerations when measuring myocardial perfusion reserve by cardiovascular magnetic resonance using regadenoson. J Cardiovasc Magn Reson. 2012;14:89.

55. Jerosch-Herold M, Seethamraju RT, Swingen CM, Wilke NM, Stillman AE. Analysis of myocardial perfusion MRI. J Magn Reson Imaging. 2004; 19:758-770.

56. Jerosch-Herold M. Quantification of myocardial perfusion by cardiovascular magnetic resonance. J Cardiovasc Magn Reson. 2010;12:57.

57. Mordini FE, Haddad T, Hsu LY, et al. Diagnostic accuracy of stress perfusion CMR in comparison with quantitative coronary angiography: fully quantitative, semiquantitative, and qualitative assessment. JACC Cardiovasc Imaging. 2014;7:14-22.

58. Patel AR, Antkowiak PF, Nandalur KR, et al. Assessment of advanced coronary artery disease: advantages of quantitative cardiac magnetic resonance perfusion analysis. J Am Coll Cardiol. 2010;56:561-569.

59. Tsaftaris SA, Zhou X, Tang R, Li D, Dharmakumar R. Detecting myocardial ischemia at rest with cardiac phase-resolved blood oxygen level-dependent cardiovascular magnetic resonance. Circ Cardiovasc Imaging. 2013;6:311-319.

60. Jahnke C, Gebker R, Manka R, Schnackenburg B, Fleck E, Paetsch I. Navigator-gated $3 \mathrm{~d}$ blood oxygen level-dependent CMR at 3.0-t for detection of stress-induced myocardial ischemic reactions. JACC Cardiovasc Imaging. 2010;3:375-384.

61. Manka R, Wissmann L, Gebker R, et al. Multicenter evaluation of dynamic three-dimensional magnetic resonance myocardial perfusion imaging for the detection of coronary artery disease defined by fractional flow reserve. Circ Cardiovasc Imaging. 2015;8:e003061.

62. Manka R, Paetsch I, Kozerke S, et al. Whole-heart dynamic threedimensional magnetic resonance perfusion imaging for the detection of coronary artery disease defined by fractional flow reserve: determination of volumetric myocardial ischaemic burden and coronary lesion location. Eur Heart J. 2012;33:2016-2024.

63. Tarroni G, Corsi C, Antkowiak PF, et al. Myocardial perfusion: nearautomated evaluation from contrast-enhanced MR images obtained at rest and during vasodilator stress. Radiology. 2012;265:576-583.

64. Lancellotti P, Nchimi A, Delierneux C, et al. Biological effects of cardiac magnetic resonance on human blood cells. Circ Cardiovasc Imaging. 2015;8:e003697.

65. Kaufmann PA. Cardiac magnetic resonance imaging: the case for nonionizing radiation protection and the precautionary principle. Circ Cardiovasc Imaging. 2015;8:e003885.

66. Schwitter J, Wacker CM, van Rossum AC, et al. MR-IMPACT: comparison of perfusion-cardiac magnetic resonance with single-photon emission computed tomography for the detection of coronary artery disease in a multicentre, multivendor, randomized trial. Eur Heart J. 2008;29:480-489.

67. Schwitter J, Wacker CM, Wilke N, et al; MR-IMPACT Investigators. MR-IMPACT II: Magnetic Resonance Imaging for Myocardial Perfusion Assessment in Coronary artery disease Trial: perfusion-cardiac magnetic resonance vs single-photon emission computed tomography for the detection of coronary artery disease: a comparative multicentre, multivendor trial. Eur Heart J. 2013;34:775-781. 
68. Freed BH, Narang A, Bhave NM, et al. Prognostic value of normal regadenoson stress perfusion cardiovascular magnetic resonance. J Cardiovasc Magn Reson. 2013;15:108.

69. Pontone G, Andreini D, Bertella E, et al. Prognostic value of dipyridamole stress cardiac magnetic resonance in patients with known or suspected coronary artery disease: a mid-term follow-up study. Eur Radiol. Epub 2015 Oct 29.

70. Patel AR, Bhave NM, Mor-Avi V. Myocardial perfusion imaging with cardiac computed tomography: state of the art. J Cardiovasc Transl Res. 2013;6:695-707.

71. Varga-Szemes A, Meinel FG, De Cecco CN, Fuller SR, Bayer RR, Schoepf UJ. CT myocardial perfusion imaging. AJR Am J Roentgenol. 2015;204:487-497.

72. Ko BS, Cameron JD, Meredith IT, et al. Computed tomography stress myocardial perfusion imaging in patients considered for revascularization: a comparison with fractional flow reserve. Eur Heart J. 2012;33:67-77

73. Iwasaki K, Matsumoto T. Myocardial perfusion defect in patients with coronary artery disease demonstrated by 64-multidetector computed tomography at rest. Clin Cardiol. 2011;34:454-460.

74. Kachenoura N, Gaspar T, Lodato JA, et al. Combined assessment of coronary anatomy and myocardial perfusion using multidetector computed tomography for the evaluation of coronary artery disease. Am J Cardiol. 2009;103:1487-1494.

75. Gupta M, Kadakia J, Jug B, Mao SS, Budoff MJ. Detection and quantification of myocardial perfusion defects by resting single-phase 64-slice cardiac computed tomography angiography compared with SPECT myocardial perfusion imaging. Coron Artery Dis. 2013;24:290-297.

76. Rochitte CE, George RT, Chen MY, et al. Computed tomography angiography and perfusion to assess coronary artery stenosis causing perfusion defects by single photon emission computed tomography: the core320 study. Eur Heart J. 2014;35:1120-1130.

77. Mor-Avi V, Lodato JA, Kachenoura N, et al. Quantitative threedimensional evaluation of myocardial perfusion during regadenoson stress using multidetector computed tomography. J Comput Assist Tomogr. 2012;36:443-449.

78. Bhave NM, Mor-Avi V, Kachenoura N, et al. Analysis of myocardial perfusion from vasodilator stress computed tomography: does improvement in image quality by iterative reconstruction lead to improved diagnostic accuracy? J Cardiovasc Comput Tomogr. 2014;8:238-245.
79. Cohn PF, Fox KM, Daly C. Silent myocardial ischemia. Circulation. 2003;108:1263-1277.

80. Winchester DE, Zhang P, Jadhav MP, Beyth RJ. A cohort study of myocardial perfusion imaging in veteran patients without symptoms: contributing factors and results of testing. Medicine (Baltimore). 2015;94:e1154.

81. Zellweger MJ, Fahrni G, Ritter M, et al; BASKET Investigators. Prognostic value of "Routine" Cardiac stress imaging 5 years after percutaneous coronary intervention: the prospective long-term observational basket (basel stent kosteneffektivitäts trial) late imaging study. JACC Cardiovasc Interv. 2014;7:615-621.

82. Kishi S, Magalhães TA, Cerci RJ, et al. Total coronary atherosclerotic plaque burden assessment by ct angiography for detecting obstructive coronary artery disease associated with myocardial perfusion abnormalities. J Cardiovasc Comput Tomogr. Epub 2016 Jan 14.

83. Otsuka K, Fukuda S, Tanaka A, et al. Prognosis of vulnerable plaque on computed tomographic coronary angiography with normal myocardial perfusion image. Eur Heart $J$ Cardiovasc Imaging. 2014; $15: 332-340$

84. Alie N, Eldib M, Fayad ZA, Mani V. Inflammation, atherosclerosis, and coronary artery disease: PET/CT for the evaluation of atherosclerosis and inflammation. Clin Med Insights Cardiol. 2014;8:13-21.

85. Sun ZH, Rashmizal H, Xu L. Molecular imaging of plaques in coronary arteries with PET and SPECT. J Geriatr Cardiol. 2014;11:259-273.

86. Wolk MJ, Bailey SR, Doherty JU, et al; American College of Cardiology Foundation Appropriate Use Criteria Task Force. ACCF/AHA/ ASE/ASNC/HFSA/HRS/SCAI/SCCT/SCMR/STS 2013 multimodality appropriate use criteria for the detection and risk assessment of stable ischemic heart disease: A report of the American College of Cardiology Foundation Appropriate Use Criteria Task Force, American Heart Association, American Society of Echocardiography, American Society of Nuclear Cardiology, Heart Failure Society of America, Heart Rhythm Society, Society for Cardiovascular Angiography and Interventions, Society of Cardiovascular Computed Tomography, Society for Cardiovascular Magnetic Resonance, and Society of Thoracic Surgeons. JAm Coll Cardiol. 2014;63:380-406.
Research Reports in Clinical Cardiology

\section{Publish your work in this journal}

Research Reports in Clinical Cardiology is an international, peerreviewed, open access journal publishing original research, reports, editorials, reviews and commentaries on all areas of cardiology in the clinic and laboratory. The manuscript management system is completely online and includes a very quick and fair peer-review system.

\section{Dovepress}

Visit http://www.dovepress.com/testimonials.php to read real quotes from published authors. 